\title{
Eficacia del uso del Tadalafilo tópico en el tratamiento de la fisura anal. Resultados de un estudio cooperativo
}

\author{
Hugo A. Amarillo, ${ }^{1}$ Eduardo J. Vaccarezza, ${ }^{2}$ Paula Casares, ${ }^{1}$ Luis Montilla ${ }^{1}$ \\ ${ }^{1}$ Sanatorio Modelo Tucumán. San Miguel de Tucumán, Argentina. \\ ${ }^{2}$ Centro Médico Alberti. Buenos Aires, Argentina.
}

\begin{abstract}
RESUMEN
Introducción: El tratamiento médico de la fisura anal crónica tiene múltiples opciones. El Tadalafilo casi no fue analizado en la bibliografía como opción terapéutica.

Objetivo: Evaluar la seguridad y efectividad del uso de Tadalafilo tópico para el tratamiento médico de la fisura anal.

Diseño: Estudio prospectivo no randomizado.

Pacientes y método: Ochenta y seis pacientes (61 \% varones). Periodo: 2011-2018. Estudio biinstitucional, centros privados. Se analizaron pacientes con fisura anal crónica de más 8 semanas de duración. Exclusión: pacientes que no finalizaron tratamiento. Se registraron resultados, efectos adversos, tiempo de tratamiento y su relación con respuesta.

Resultados: Se estudiaron 86 pacientes, 53 varones (61\%), 77 de ellos mayores a 55 años. El 82,5 \% logró la curación y el $11,6 \%$ fue sometido a cirugía, los restantes cronificaron su tratamiento médico con buena respuesta clínica. El $72 \%$ hizo 2 meses de tratamiento ( $\mathrm{p}=0,002) ; 7$ pacientes, 3 meses; 12, entre 3 y 6 meses; y 5 , más de 6 meses. El $11 \%$ fue sometido a cirugía $(p=0,54)$. El $64 \%$ no presentó patología anal previa y el resto no modificó sus resultados a pesar de la asociación. El $95 \%$ no presentó ningún efecto adverso y los relatados fueron leves (rush, trombosis, sangrado leve).

Conclusión: El tratamiento con Tadalafilo presenta un elevado índice de curación y resolución sintomática con casi nulos efectos secundarios. En los casos con falla, la cirugía resolvió dicha patología. Otros estudios comparativos con otros métodos farmacológicos y/o cirugía podrían afirmar estos resultados.
\end{abstract}

Palabras claves: Fisura anal; Tadalafilo; Esfinterotomía; Nitritos

\begin{abstract}
Introduction: The medical treatment of chronic anal fissure has multiple options. Tadalafil was hardly analyzed in the literature as a therapeutic option.

Objective: To evaluate the safety and effectiveness of the use of topical Tadalafil for the medical treatment of anal fissure. Design: Observational non-randomized prospective study.

Patients and methods: Patients with chronic anal fissure lasting more than 8 weeks treated with Tadalafil during the 20112018 period were analyzed. Those who did not complete treatment were excluded. Cure rate, adverse effects, treatment time and its relationship with response were recorded.

Setting: Two private medical institutions.

Results: Eighty-six patients, 53 men (61\%), 77 of them older than 55 years, were analized. Cure was achieved by $82.5 \%$ of patients, $11.6 \%$ underwent surgery, and the remaining patients continue chronic medical treatment with a good clinical response. Seventy-two percent of patients had 2 months of treatment ( $p=0.002) ; 7$ patients, 3 months; 12 , between 3 and 6 months; and 5, more than 6 months. Sixty-four percent of patients did not present previous anal pathology and the rest did not modify their results despite the association. No adverse was presented in 95\% of patients and those reported (rush, thrombosis, light bleeding) were mild.

Conclusion: Treatment with Tadalafil has a high cure rate and symptomatic resolution with almost no side effects. In failure cases surgery resolved the condition. The performance of other comparative studies with other pharmacological methods and/ or surgical treatment could affirm these results.
\end{abstract}

Keywords: Anal fissure; Tadalafil; Sphincterotomy; Nitrites

\section{INTRODUCCIÓN}

La fisura anal es una de las patologías más frecuentes de la consulta proctológica y, a pesar de que se dispone de evidencia suficiente y numerosas posibilidades terapéuticas, el tratamiento es aún desafiante, en algunos casos controversial y un número no menor de pacientes son refractarios al mismo. ${ }^{1,2}$

La fisura anal es una lesión dolorosa de la región anal que se presenta como ulceración del rafe, habitualmente posterior. Es aguda cuando no se extiende más allá de las

Hugo Amarillo

bugoamarillo@gmail.com

Recibido: mayo de 2020. Aceptado: septiembre de 2020

Los autores declaran no tener ningún conflicto de interés.
6 a 8 semanas. Luego de dicho período se considera como crónica, asociada a la persistencia de los síntomas (síndrome fisurario) y los hallazgos físicos de la papila hipertrófica, hemorroides centinela y la visualización del esfínter anal interno. ${ }^{3,4}$

Dada la posible discapacidad asociada con la cirugía, el futuro mediato con el riesgo consecuente de alteraciones de la continencia, nuevas alternativas médicas de tratamiento son puestas en consideración. ${ }^{2-5} \mathrm{El}$ uso del Tadalafilo planteado como opción terapéutica no ha sido suficientemente estudiado. El uso de inhibidores de la 5 fosfodiesterasa para lograr la curación de la fisura anal es reciente y no registra antecedentes a nivel nacional. ${ }^{6}$

El Tadalafilo es un potente inhibidor selectivo de la fosfodiesterasa. Se ha demostrado in Vitro que la fosfodies- 
terasa 5 es la responsable de la degradación del GMP cíclico dentro de la célula muscular lisa, la inhibición de la misma por parte del Tadalafilo se traduce la persistencia de niveles elevados de GMP cíclico dentro de la célula, con la consecuente relajación del esfínter anal. Adicionalmente, ante un óptimo estado de funcionamiento, la célula muscular lisa es capaz de sintetizar colágeno tipo III, proteoglicanos y elastina que promueven la cicatrización. ${ }^{6}$

El objetivo del presente trabajo fue evaluar la seguridad y efectividad del uso de Tadalafilo tópico para el tratamiento médico de la fisura anal.

\section{MATERIAL Y MÉTODOS}

En un estudio prospectivo no randomizado, entre dos instituciones privadas (una de ellas universitaria), se registraron, en el periodo comprendido entre julio de 2011 y julio de 2018, todos los pacientes que consultaron en forma espontánea con los siguientes criterios de inclusión:

- diagnóstico de fisura anal evidente al examen físico y

- tiempo de duración mayor o igual a 8 semanas.

No se consideró como criterio de exclusión pacientes con tratamiento médico o quirúrgico previo o patología orificial asociada. Se excluyeron los pacientes que no pudieron o no completaron el tratamiento por causas no relacionadas al tratamiento.

Se registraron en forma consecutiva que cumplían con los criterios de selección en una base de datos planilla de cálculos Excel Microsoft. Los datos registrados fueron: edad, género, resultado del tratamiento, tiempo de tratamiento y presencia de cirugía previa. Asimismo, se registraron los efectos adversos con el principio químico o complicaciones asociadas.

Las variables de estudio se definieron en:

- Resultado de tratamiento con Tadalafilo que se definió como

- curación: cura definitiva con tratamiento médico,

- cronificación: uso de tratamiento médico por persistencia de enfermedad,

- necesidad de cirugía para curar patología por falta de respuesta.

- Tiempo de tratamiento con Tadalafilo que se definió desde el inicio del tratamiento hasta la finalización del mismo en:

- 1 mes,

- 2 meses,

- 3 meses y

- más de 3 meses.

- Patología perianal previa que se definió como:

- ninguna: sin antecedentes patológicos,

- hemorroides, fístula, etc.,

- cirugía previa por fisura: antecedente de trata- miento quirúrgico previo de tratamiento de fisura anal.

Todos los pacientes fueron evaluados clínicamente antes del tratamiento y durante su seguimiento, el cual fue mensual durante 6 meses y luego anual.

Lugar: sector de Coloproctología del Sanatorio Modelo de Tucumán, entre 2015 y 2019, y del Centro Médico Alberti, Buenos Aires.

\section{Fórmula}

La fórmula magistral crema tópica de Tadalafilo aplicada 3 veces al día. Dicha fórmula fue elaborada en forma magistral a nivel local y homogénea entre ambos centros, y basada en la fórmula propuesta por Alfonso et al., ${ }^{6} \mathrm{y}$ consistió en una mezcla de:

- Poliéster Mucopolisacárido del ácido sulfúrico (órgano heparinoide) $4 \mathrm{mg}$,

- prednisolona $1 \mathrm{mg}$,

- oxipolietoxidodecano $50 \mathrm{mg}$,

- hexaclorafeno $5 \mathrm{mg}$,

- clorhidrato de lidocaína al $2 \%$ y

- Tadalafilo $40 \mathrm{mg}$.

\section{RESULTADOS}

Se estudiaron un total de 86 pacientes con diagnóstico de fisura anal, siendo 53 del sexo masculino (61\%) y 33 del sexo femenino (Gráfico 1).

En lo correspondiente al resultado del tratamiento con Tadalafilo son expresados en el Gráfico 3. El 82,5 \% (71) de los pacientes lograron curación, mientras que 10 (11,6 \%) tuvo que ser sometido a cirugía para lograr su curación por falla en la respuesta al tratamiento con Tadalafilo. Los 5 pacientes restantes $(5,8 \%)$ cronificaron su tratamiento médico, aunque con buena respuesta y desaparición de los síntomas por el tratamiento. Estos fueron considerados por separado de la curación, a pesar de que pudieron obviar la cirugía como tratamiento final. De incluir todos los casos con respuesta favorable a los síntomas con el tratamiento con Tadalafilo fue del $88 \%$.

En relación al tiempo de tratamiento con Tadalafilo (Gráfico 4), se observó que hicieron al menos 2 meses de tratamiento el $72 \%(62)$ de los ( $\mathrm{p}=0,002)$, mientras que 7 pacientes hicieron 3 meses, 12 (14\%) pacientes hicieron tratamiento entre 3 y 6 meses y 5 pacientes más de 6 meses.

Al analizar la relación entre el tiempo de tratamiento y su resultado, se observó que: el $83 \%$ de los pacientes que se curaron necesitaron 2 meses de tratamiento $(\mathrm{n}=59)$ y 3 de ese grupo requirió de cirugía. De los 7 pacientes que hicieron 3 meses, fue necesario operar a 2 pacientes, mientras que 5 de los pacientes que se trató entre 3 y 6 meses fueron operados; el $6 \%$ cronificó. E1 $11 \%$ fue so- 
metido a cirugía $(\mathrm{p}=0,54)$ (Gráfico 5).

Para la variable patología perianal previa, el $64 \%$ no presentó patología previa, mientras que la patología hemorroidal fue la más prevalente (17\%) entre los pacientes con antecedentes, sin encontrar significancia estadística.

Para la variable de efectos adversos el tratamiento con Tadalafilo, se observó que el 95 \% (77) no presentó ningún efecto adverso, mientras que solo 4 pacientes presentaron efectos adversos: 2 pacientes presentaron rush cutáneo que cedió con la suspensión progresiva del tratamiento, un paciente presentó trombosis externa y 1 paciente con sangrado leve. No hubo ningún efecto cardiovascular central o periférico ni alteraciones de la continencia de ningún tipo (Gráfico 6).

El seguimiento fue mensual durante los primeros 6 meses y luego anual, hasta los 2 años. Se pudo lograr dicho seguimiento en el $91 \%$ de los casos (2 años), donde no se registró recurrencia precoz luego de lograr la curación. En 2 casos hubo recurrencia luego de los 2 años y requirieron de cirugía para su tratamiento.

\section{DISCUSIÓN}

El tratamiento de la fisura anal se basa en la institución de medidas higiénico-dietéticas, métodos farmacológicos que relajan el músculo lisotales como: pomadas a base de óxido nítrico (neurotransmisor que impide la contracción muscular), bloqueadores de canal de calcio (nifedipina y diltiazem) o la aplicación de toxina botulínica. ${ }^{1,2,-10}$

Algunos de estas drogas se asocian a diversos efectos adversos como ser: cefalea, lipotimia, mareos e hipotensión arterial (nitroglicerina y bloqueantes cálcicos), llevando al abandono del tratamiento por parte de los pacientes. ${ }^{3-5}$

En una revisión Cochrane, el tratamiento médico con nitritos o bloqueadores cálcicos o con Toxina Botulínica tiene mejores resultados que el placebo, aunque con menor efectividad que la cirugía. Los nitritos curan hasta un $49 \%$ con una recurrencia del 50 al $60 \%$. La eficiencia de la toxina botulínica es del $67,5 \% .^{2}$

Con respecto a la toxina botulínicano se encontró una asociación clara entre la dosis, la preparación, el sitio de inyección y las tasas de curación, sumado al elevado costo del tratamiento y la posibilidad igual o mayor al $60 \%$ luego de interrumpir el tratamiento. ${ }^{11}$

La nifedipina es superior a los nitritos en sus resultados $\mathrm{y}$ con menores efectos secundarios e interacciones medicamentosas. ${ }^{7,8}$

La tasa de cicatrización de la esfinterotomía lateral es entre el 92 y $100 \%$ con incontinencia del 3,3 al 16\%.,10

El tratamiento médico presenta menos consecuencias que la cirugía (recomendación 1B). Por ello y por su seguridad, representa el tratamiento de primera línea (reco-

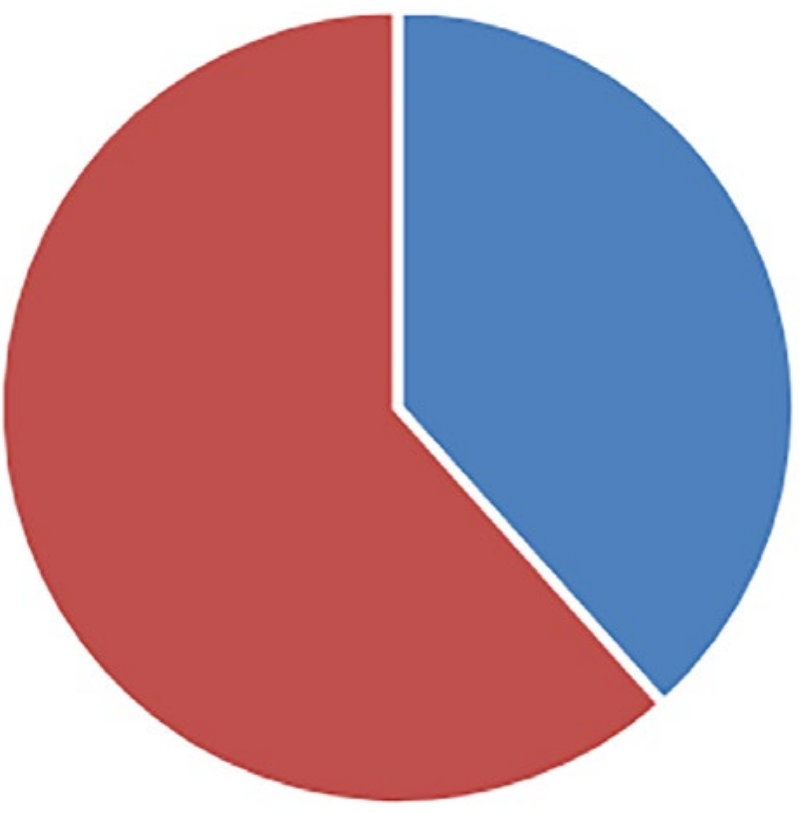

\section{Femenino Masculino}

Gráfico 1: Distribución por género.

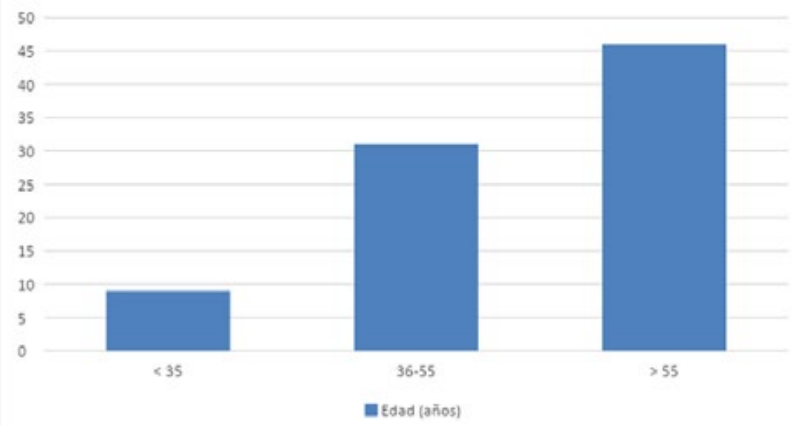

Gráfico 2: Distribución por edad.

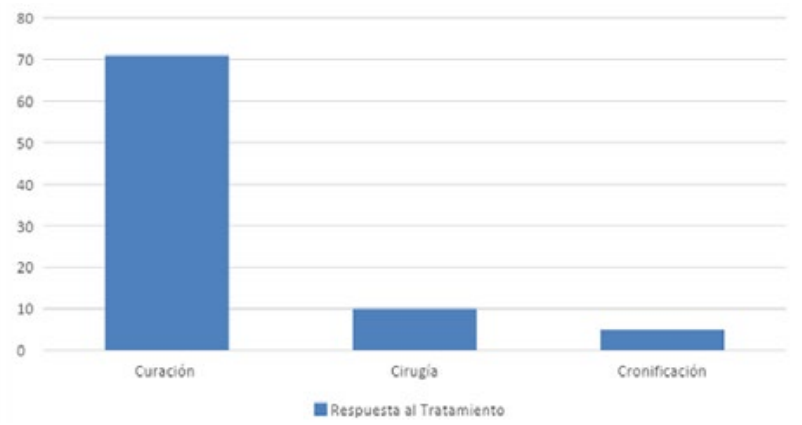

Gráfico 3: Resultados del tratamiento.

mendación 1B). ${ }^{5}$ Los bloqueantes de los canales de calcio tiene menores efectos secundarios y constituyen la primera línea (recomendación $1 \mathrm{~A}){ }^{5}$

Existe casi nula bibliografía respecto al uso del Tadalafilo tópico en el tratamiento de la fisura anal. Actualmente no está disponible comercialmente de aplicación tópica, por lo que debe realizarse una fórmula magistral, la que fue implementada según la estructura farmacológica pro- 


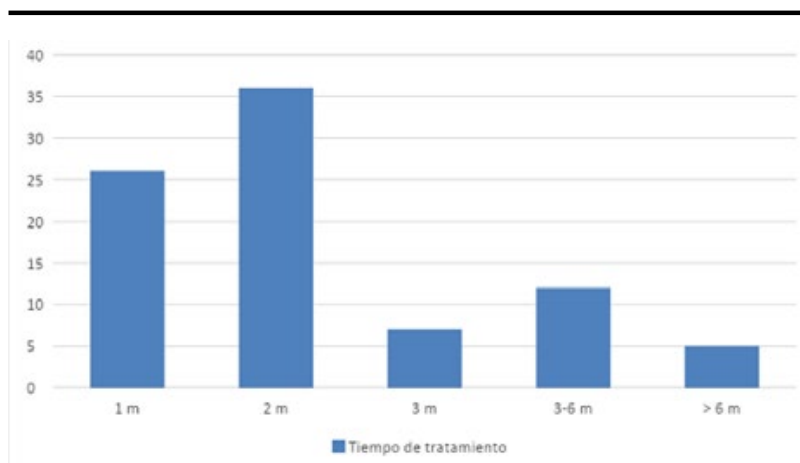

Gráfico 4: Tiempo de tratamiento.

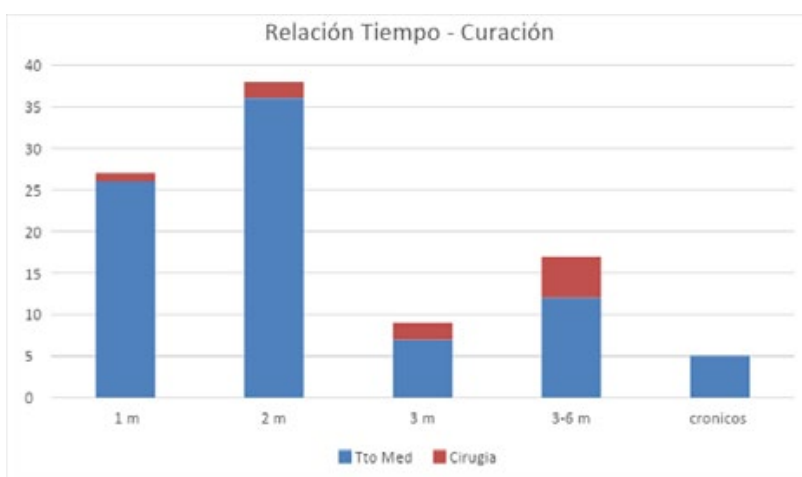

Gráfico 5: Relación de tiempo de tratamiento y resultado.

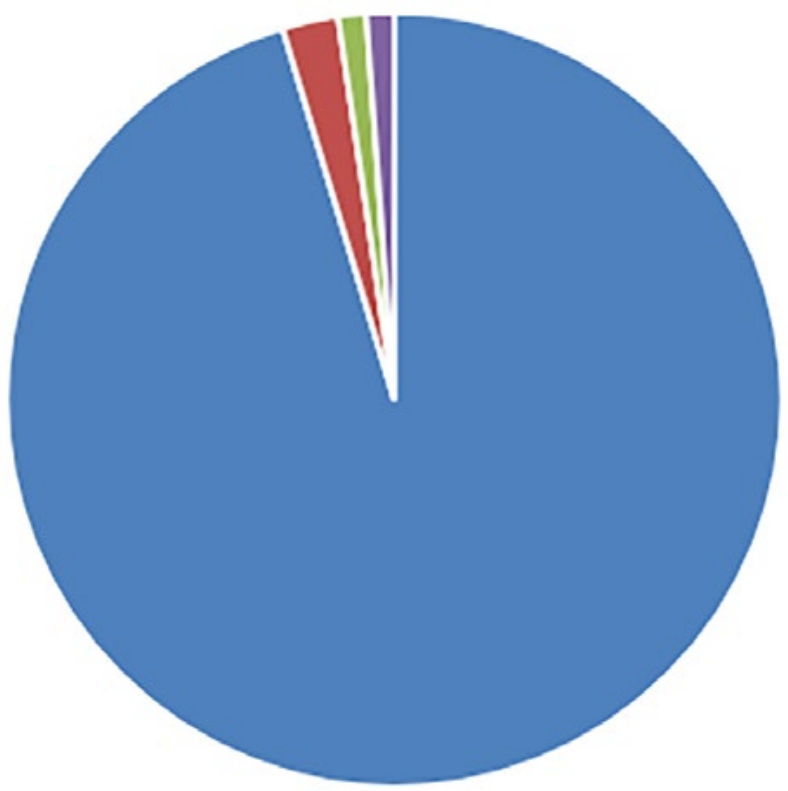

Sin efectos $\square$ Rush

Gráfico 6: Efectos adversos del tratamiento con Tadalafilo.

puesta por Alfonso et al., ${ }^{6} \mathrm{y}$ cuyos resultados pudimos reproducir. En el mercado farmacéutico extranjero, existe el Tadalafilo como producto oral o jalea tópica, pero no se encontró evidencia sobre Tadalafilo tópico como presentación para el tratamiento de la fisura a excepción del trabajo mencionado.

Nuestra serie demostró que el 83 \% de los pacientes que utilizaron Tadalafilo en un tiempo promedio de 2 meses logró la curación de su patología. Si consideramos al grupo que mejoró su cuadro clínico, pero cronificó su tratamiento por múltiples motivos, el índice llegó casi al $88 \%$, constituyendo en estos casos una opción para pacientes con contraindicación de cirugía.

En correlación al trabajo de Núñez et al., publicado en el año 2012, demostramos respuesta satisfactoria con el uso de Tadalafilo en un índice elevado. ${ }^{6}$ Se demostró en este estudio que un $10 \%$ de pacientes requirió de cirugía para su resolución, un índice levemente superior a las series relatadas por cada uno de los centros participantes de este estudio por separado.

Lamentablemente, no existen estudios comparativos del Tadalafilo con la esfinterotomía lateral o con otros tratamientos médicos. En esta serie, el diseño no comparativo se basa en el elevado índice de efectos secundarios observados con otras drogas (nitritos) y la baja tasa de respuesta asociada a estos y a las cremas comunes. Del mismo modo, la cirugía solo se indica ante el fracaso de la terapia médica.

En el análisis del tiempo de tratamiento y su relación con su resultado, no se encontró evidencia significativa, por lo que una nueva línea de investigación imprime la necesidad de obtener una muestra mayor de pacientes con diagnóstico de fisura anal que utilicen Tadalafilo como tratamiento de primera línea, ya que nuestra experiencia reflejó que su uso fue favorable para la curación de la enfermedad como terapia de primera línea. Sería, asimismo, necesario comparar la eficacia de otros fármacos considerados como primera línea con el Tadalafilo.

La limitación de esta serie, si bien incluye experiencias de dos instituciones distantes con poblaciones y resultados similares, es que su población es pequeña y la falta de comparación con otros métodos, tanto médicos como quirúrgicos.

La toxina botulínica es similar al tratamiento médico con mejor cicatrización y constituye la segunda línea (recomendación 1 C). ${ }^{5,8-10}$

La esfinterotomía es más eficaz que la toxina botulínica a los 3 años, a pesar de una incontinencia del $9 \%$ con una recurrencia de la toxina botulínica de hasta el $50 \%$ a los 3 años. ${ }^{11}$

Los efectos secundarios de los nitritos (cefalea) y la incontinencia asociada a la cirugía, a pesar de su alta efectividad y de algunos reportes de incontinencia transitoria secundaria a la toxina botulínica, deben ser considerados. Sin embargo, y al igual que Nuñez, el tratamiento es efectivo en un alto porcentaje, de difícil acceso por su alto costo de producción, pero de alta adhesión y casi nulos efectos adversos. Por estos motivos en pacientes con cirugía previa por fisura o cirugía orificial, o bien con morblilidad asociada que contraindica la cirugía, el Tadalafilo podría 
ser una excelente opción a pesar de requerir de un tratamiento prolongado.

\section{CONCLUSIONES}

El tratamiento de la fisura anal crónica con Tadalafilo tó- pico es factible y presenta un elevando índice de curación. Es un método seguro, dado que no presentaron ningún efecto secundario asociado grave, ni efectos cardiovasculares.

Constituye una opción válida para pacientes con contraindicación de cirugía o alteraciones de la continencia.

\section{REFERENCIAS}

1. Arroyo A, Montes E, Calderón T, Blesa I, Elía M, Salgado G, et al. Tratamiento de la fisura anal: algoritmo de actuación. Documento de consenso de la Asociación Española de Coloproctología y la Sección de Coloproctología de la Asociación Española de Cirujanos. Cir Esp 2018;96:260-67.

2. Nelson R, Thomas K, Morgan J, Jones A. Non-surgical therapy for anal fissure. Review. The Cochrane Library 2012, Issue 12.

3. Latif J, Rodriguez Martin J, Sanchez I. Fisura anal, opciones terapéuticas actuales. Rev Argent Coloproct 2008;19:1-12.

4. Hequera J. Fisura anal. En: Galindo F. Cirugía Digestiva. www.sacd. org.ar. 2009;III-377:1-13.

5. Stewart DB, Gaertner W, Glasgow S, Migaly J, Feingold D, Steele SR. Clinical Practice Guideline for the management of anal fissures. Dis Colon Rectum 2017;60:7-14.

6. Alfonzo Nuñez R, Cardozo Madrid O, Garcia D, Bacarini D. Tratamiento médico de la fisura anal con Tadalafilo tópico como principio activo. Rev Argent Coloproct 2012;23: 32-5.
7. Cross KLR, Massey EJD, Fowler AL, Monson JRT. The management of anal fissure: ACPGBI position statement. Colorectal Dis 2008;10(Suppl 3):1-7.

8. Wald A, Bharucha A, Cosman B, Whitehead W. ACG clinical guideline: Management of benign anorectal disorders. Am J Gastroenterol 2014;109:1141-57.

9. Altomare D, Binda G, Canuti S, Landolfi V, Trompetto M, Villani $\mathrm{R}$. The management of patients with primary chronic anal fissure: A position paper. Tech Coloproctol 2011;15: 135-41.

10. Ebinger S, Hardt J, Warschkow R, Schmied B, Herold A, Post S, et al. Operative and medical treatment of chronic anal fissures-A review and network meta-analysis of randomized controlled trials. J Gastroenterol 2017;52:663-76.

11. Barbeiro S, Atalaia-Martins C, Marcos P, Goncalves C, Canhoto $\mathrm{M}$, Arroja B, et al. Long-term outcomes of botulinum toxin in the treatment of chronic anal fissure: 5 years of follow-up. Eur Gastroenterol J 2017;5:293-97.

\section{COMENTARIO}

Gran parte de los paciente con fisura anal resolverán el cuadro clínico sin intervención quirúrgica, solo modificando hábitos o con la implementación de tratamiento médico, sin embargo existen diferentes tipos de tratamientos y opciones quirúrgicas para aquellos que no responden.

La mayoría de los tratamientos tienen como principal objetivo actuar sobre la hipertonía esfinteriana, principal factor implicado en la fisiopatogenia de la fisura anal. Para conseguir tal efecto se han implementado formulaciones con 2 o más drogas como principios activos, combinados con dosis fijas. Está demostrado que la combinación es beneficiosa cuando los ingredientes son incorporados de manera segura y efectiva en un número significativo de pacientes, y ofrece algunas ventajas sobre la administración simultánea de diferentes preparados de un solo componente activo, ya que mejora la compliance, eficacia y reduce los efectos adversos locales y costos.

Las preparaciones tópicas que contienen corticoides y anestésicos locales, son extensamente utilizadas para aliviar síntomas en diferentes patologías, especialmente la patología anal.

Los corticoides actúan mediante la unión a receptores esteroides reduciendo la síntesis de mediadores inflamatorios, la dilatación de capilares y el edema, por lo cual la mejora de los síntomas no es inmediata. En cambio, los anestésicos locales proveen alivio inmediato del dolor luego de su aplicación por lo que se logra un efecto sinérgico con la combinación de ambos componentes.

Si a esta combinación se le suma un principio activo como el tadalafilo (utilizado ampliamente para patología urológica), podría generar un mayor beneficio al actuar sobre la relajación de la musculatura esfinteriana, pero no puede atribuírsele directamente el éxito de la cura de la fisura

Si bien es una limitante del trabajo descripta por los autores, se aguardan trabajos que demuestren el efecto de la droga como único principio activo, esto podría lograrse con un grupo control.

Romina Bianchi

Hospital José María Penna y Hospital Universitario Fundación Favaloro, Ciudad Autónoma de Buenos Aires, Argentina 